\title{
Wave pattern induced by a moving atmospheric pressure disturbance
}

\author{
Xiaojing Niu*, Haojie Zhou \\ State Key Laboratory of Hydroscience and Engineering, \\ Department of Hydraulic Engineering, Tsinghua University, Beijing, China
}

\begin{abstract}
A moving low atmospheric pressure is a main feature of tropical cyclones, which can induce a system of forced water waves and is an important factor that cause water level rise during a storm. A numerical model based on the nonlinear shallow water equation is applied to study the forced waves caused by an atmospheric pressure disturbance moving with a constant velocity over water surface. The effects of the moving speed, the spatial scale and the central pressure drop of the pressure disturbance are discussed. The results show that the wave pattern caused by a moving low-pressure is highly related with its moving speed. The wave pattern undergoes a great change as the moving speed approaches the wave velocity in shallow water. When the moving speed is less than the wave velocity, the distribution of water surface elevation is nearly the same as that of the pressure disturbance, and the maximum of the water surface elevation is located at the center of pressure. When the moving speed is larger than the wave velocity, a triangle shaped wave pattern is formed with a depression occurs in front of the pressure center, and the maximum of the water surface elevation lags behind the center of pressure. As the moving speed increases, the maximum of the water surface elevation firstly increases and then decreases, which reaches a peak when the moving speed is close to the wave velocity. The maximum of water surface elevation is approximately in proportion to the central pressure drop, and slightly affected by the spatial scale of pressure disturbance. Both the central pressure drop and the spatial scale of the pressure disturbance do not significantly affect the forced wave pattern. However, a clear difference can be noticed on the ratio of the maximum water surface elevation in moving pressure situation to that in static situation, when the moving speed is close to the wave
\end{abstract}

${ }^{*}$ Corresponding author. Address: Department of Hydraulic Engineering, Tsinghua University, Beijing, 100084,

China; Tel: +86-10-62797367; Email: nxj@tsinghua.edu.cn

$1 / 10$ 
velocity. A pressure disturbance with smaller spatial scale and smaller central pressure drop will give a larger ratio when the moving speed is close to the wave velocity.

Keywords: water level rise; wave pattern; moving atmospheric pressure disturbance; nonlinear shallow water model

\section{Introduction}

Physical mechanism of storm surge has long been a major concern in coastal engineering, which is one of most important issues in accurately predicting storm disaster. Storm surge is a tsunami-like phenomena of water level rise, which is induced by coupling effects of strong wind, low atmospheric pressure, water waves and heavy rain in tropical cyclones. The low atmospheric pressure is a main feature of tropical cyclones, which is one of the most important factors that cause the water level rise. In history, a sea-level pressure as low as $870 \mathrm{hPa}$ was recorded during the Typhoon Tip on Oct. 12, 1972, the strongest tropical cyclones ever recorded.

Water wave caused by atmospheric pressure disturbance is an interesting topic, but researches on this topic are rare. Early in 1936, Inui ${ }^{[1]}$ derived an analytical solution on the water wave induced by a moving pressure disturbance, on the assumption that both the water depth and the dimention of disturbance are finite. Greenspan (1956) ${ }^{[2]}$ analytically studied the edge wave induced by a pressure disturbance moving along the coastline. Abraham (1963) ${ }^{[3]}$ carried out a series of laboratorial experiment on water waves induced by a moving pressure. Mercer et al. (2002) ${ }^{[4]}$ numerically studied the wave induced by a quickly moving pressure disturbance. Vennell ${ }^{[5-7]}$ published a series of analytical studies on the resonance and trap of waves induced by a moving pressure in a shelf. An et al. (2012) ${ }^{[8]}$ and Seo et al. (2014) ${ }^{[9]}$ provided a numerical study and a new analytical solution on the edge wave induced by a pressure disturbance moving along coastline.

It is known that decrease of pressure with $1 \mathrm{hPa}$ will lead to $1 \mathrm{~cm}$ elevation of water level under static conditions. However, in most cases the low atmospheric pressure weather system is moving, and its moving speed can be very fast sometimes. We analyzed the moving speed of tropical cyclones from the best track dataset of historical tropical cyclones in Western North Pacific and South China Sea. It was 
found that in over $90 \%$ situations the moving speed is between $2.5 \mathrm{~km} / \mathrm{h}$ and $37.5 \mathrm{~km} / \mathrm{h}$, but there are still more than $0.5 \%$ situations beyond $80 \mathrm{~km} / \mathrm{h}$. As known that the velocity of shallow water wave is about $36 \mathrm{~km} / \mathrm{h}$ in $10 \mathrm{~m}$ depth of water and about $113 \mathrm{~km} / \mathrm{h}$ in $100 \mathrm{~m}$ depth of water, it can be seen that the moving speed of a pressure disturbance may possibly approach or exceed the velocity of shallow water wave in shallow sea.

A moving pressure disturbance may cause much larger water level rise and a much more complex wave pattern. A simple analytical expression that describes the forced wave induced by a moving pressure disturbance in the open ocean was provided by Proudman (1929) ${ }^{[10]}$, which is well-known and still been widely used to explain meteotsunami ${ }^{[11]}$. The expression revealed a resonance phenomenon when the travelling speed of pressure disturbance is closed to the local water wave velocity, which later came to be known as "Proudman resonance". The forced waves predicted by the Proudman expression share the same spatial-temporal distribution of the atmospheric pressure disturbances. However, further researches found the wave pattern is much more complex. In this study, we focused on the forced wave induced by a moving circular low-pressure disturbance. The ideal case is abstracted from the storm surge problem. Inui's analytical solution ${ }^{[1]}$ and Abraham's experiment ${ }^{[3]}$ are the most useful references on this topic. Abraham's experiment was carried out in a towing tank and the moving low-pressure was simulated by towing a suction fan over the water surface. Due to the limitation of the experiment conditions, the ratio of the effective diameter of the pressure disturbance to the water depth in his study is 0.1 to 4. It is much smaller than that in the practical problem of a low atmospheric pressure moving over the sea surface. Inui's study was based on the three-dimensional potential flow theory and the small amplitude wave assumption, and the analytical solution was given according to the Fourier's integral theorem. Inui's analysis showed a resonance feature of the water level change induced by a moving pressure disturbance. His solution predicted that a moving low pressure can cause an infinite water level rise when the moving speed of pressure equals to the shallow water velocity. However, as Inui pointed out, the infinite rise in water level is naturally meaningless, since the analysis was based on the small amplitude wave assumption. The linear solution would lose its accuracy when the forced wave is amplified near resonance. In the near resonance cases, the nonlinear effect is significant and can not be ignored. However, analytically solving the nonlinear shallow water equation is 
quite difficult. In reality, destructive waves induced by atmospherical pressure disturbance are always the result of resonance phenomena. The maximum water level rise that may happen near resonance is of greatest concern. Nowadays, it is easy to analysis this problem by using the developed numerical model for nonlinear long waves. It provides a way to find out how the nonlinearity affects the wave pattern induced by a moving atmospheric pressure disturbance.

Based on the nonlinear shallow water model, this study focuses on the wave pattern and the maximum water level rise that induced by an idealized circular moving low-pressure. In order to get better understanding on how the maximum water level rise is affected by the parameters of a low-pressure disturbance, a series of idealized cases are numerically studied in this paper. The problem is simplified as a circular low-pressure disturbance acting on an infinite sea with constant water depth, without consideration of winds. The model used in this study is simply summaried in section 2. And then the effects of each parameter on the wave pattern and the maximum water surface elevation are discussed in Section 3.

\section{Method}

The nonlinear shallow water equations are adopted to simulate the water waves caused by pressure disturbance. The effects of Coriolis force, wind surface stress, wave radiation stress on the water surface fluctuations are ignored. The governing equations expressed in the Cartesian coordinates are shown as follows ${ }^{[12]}$.

$$
\begin{gathered}
\frac{\partial \eta}{\partial t}+\frac{\partial p}{\partial x}+\frac{\partial q}{\partial y}=0 \\
\frac{\partial p}{\partial t}+\frac{\partial}{\partial x}\left(\frac{p^{2}}{h}\right)+\frac{\partial}{\partial y}\left(\frac{p q}{h}\right)+g h \frac{\partial \eta}{\partial x}+\frac{h}{\rho} \frac{\partial P_{a}}{\partial x}+\frac{g p \sqrt{p^{2}+q^{2}}}{C^{2} h^{2}} \\
-h\left(\frac{\partial}{\partial x}\left[v_{e} \frac{\partial}{\partial x}\left(\frac{p}{h}\right)\right]+\frac{\partial}{\partial y}\left[v_{e} \frac{\partial}{\partial y}\left(\frac{p}{h}\right)\right]\right)=0 \\
\frac{\partial q}{\partial t}+\frac{\partial}{\partial x}\left(\frac{p q}{h}\right)+\frac{\partial}{\partial y}\left(\frac{q^{2}}{h}\right)+g h \frac{\partial \eta}{\partial y}+\frac{h}{\rho} \frac{\partial P_{a}}{\partial y}+\frac{g q \sqrt{p^{2}+q^{2}}}{C^{2} h^{2}} \\
-h\left(\frac{\partial}{\partial x}\left[v_{e} \frac{\partial}{\partial x}\left(\frac{q}{h}\right)\right]+\frac{\partial}{\partial y}\left[v_{e} \frac{\partial}{\partial y}\left(\frac{q}{h}\right)\right]\right)=0
\end{gathered}
$$

Where $t$ is time; $\eta$ is the surface elevation; $p$ and $q$ are the depth-averaged flux per 
unit width in $x$ and $y$ direction; $h$ is the total water depth, $h=d+\eta$, here $d$ is the still water depth; $g$ is the gravity acceleration; $\rho$ is the water density. $P_{a}$ is the atmospheric pressure at sea level; $C$ is the Chezy resistance coefficient; $v_{e}$ is the eddy viscosity. Eq. (1) is the conservation of mass integrated over the vertical. Eqs.(2) and (3) are the conservation of momentum integrated over the vertical, in which the fifth terms represent the forces exerted by the atmospheric pressure, the sixth terms are the bottom friction, and the last terms represent the turbulent diffusion. Governing equations are discretized in a staggered mesh by finite volume method, and the discrete equations are solved by ADI (Alternating Direction Implicit) method. This numerical scheme has been proved efficient and of good stability ${ }^{[12]}$.

To simplify the complexity of the practical problem, we assume that a moving pressure disturbance is acting on an infinite sea with constant water depth. The pressure disturbance used in this study is circular, and moves with a constant speed $U$. As the center of the pressure disturbance is located at $x=0$ and $y=U t$, the pressure at a particular location is given as

$$
P_{a}=P_{n}-\Delta P\left[1-\exp \left(-\frac{R_{m}}{\sqrt{x^{2}+(y-U t)^{2}}}\right)\right]
$$

Where, $P_{n}$ is the neutral pressure; $\Delta P$ is the maximum pressure drop, which is the difference between the neutral pressure and the pressure in the center of the low pressure system; $R_{m}$ is defined as the radius of pressure disturbance.

The initial-value problems are computed in our study. Velocity and surface elevation all over the computational domain are set to be zero as the initial condition. In order to avoid the computational instability due to a sudden pressure drop, the maximum pressure drop is ramped up in time with a ramp function $F t=1-e^{-t / t_{0}}$, where $t_{0}=2 \mathrm{~h}$ is employed in all the cases. The water surface elevation at the boundary is set to be zero. The domain size is chosen to be big enough to avoid boundary reflection. Generally, the domain size in both $\mathrm{x}$ and $\mathrm{y}$ direction is set to be $100 R_{m}$. The mesh sizes $\Delta x=\Delta y=R_{m} / 20$ are employed. 


\section{Results and discussion}

\subsection{Impact of the moving speed on wave pattern}

The water wave induced by a moving pressure disturbance is more complicated than that induced by a static pressure disturbance. In this section, the water waves induced by pressure disturbances moving along $x=0$ with different speed are numerically simulated. All the other parameters are fixed. The water depth remains constant as $10 \mathrm{~m}, R_{m}$ is fixed to be $40 \mathrm{~km}$ and the center pressure is set to be $20 \mathrm{hPa}$ lower than the neutral pressure. The numerical results show that the water surface elevation observed in the coordinates moving with the pressure disturbance is independent of time after the pressure drop is ramped up to its target value. Figure 1 shows the profile of water surface elevation along $x=0$ in the case of $U=c$ at different time, and it can be seen that the surface elevation increases with time and finally reachs a steady result. The numerical results after the computations reach steady states are discussed in the following section.

Numerical results show a clear difference in the wave pattern under different pressure moving speed, which have a good correlation with the ratio of the pressure moving speed to the phase velocity of shallow water waves. Figure 2 shows the wave pattern under the cases with moving speed $U=0,0.5 c, 1.0 c, 2.0 c$. In which $c=\sqrt{g d}$ is the phase velocity of shallow water waves. The origin of coordinates is defined at the center of pressure disturbance.

As the pressure moves along $x=0$, the induced waves show symmetry about $x=0$. In the static case, $U=0$, the water surface elevation is in direct proportion with the local pressure drop. Therefore, the distribution of water surface elevation is the same as the form of the pressure disturbance, which is circular with a maximum at the center. In the case of $U=0.5 c$, the water surface elevation becomes an elliptical shape and the maximum value increases. In the case of $U=1.0 c$, a water level depression appears in front of the pressure center, and obvious increase in water surface elevation occurs at the rear side of the pressure center. It is observed that the contour becomes compact ahead the pressure center and become loosened behind the pressure center, while the maximum water surface elevation increases with the increase of moving speed, as $U$ increases from 0 to 1.0 c. In the case with $U=2.0 c$, a triangle shaped wave pattern is formed, and the affected area is wider than other cases. However, the 
maximum water surface elevation grows down comparing to $U=1.0 \mathrm{c}$.

To compare the values of water level rise in each case, the water surface elevation along $x=0$ is drawn in Figure 3. In which $\eta_{0}$ is the maximum analytical value in static case, $\eta_{0}=\Delta P / \rho g$. In all cases, the maximum water level occurs near the center of pressure disturbance. When $U<c$, the position of the maximum water surface elevation is essentially coincident with the center of pressure disturbance. When $U \geq c$, a lag between the maximum water surface elevation and the center of pressure appears, and becomes larger as $U$ increases. It is clear that the maximum water surface elevation can vary greatly according to the moving speed of pressure disturbance. The variation of maximum water surface elevation is nonmonotonic with the moving speed.

A classic analytical solution has been derived by Inui (1936) ${ }^{[1]}$. The original form of his solution is complex, but a simple conclusion from the solution gives that the water surface elevation is proportional to $1 / \sqrt{1-(U / c)^{2}}$ when $U<c$ and is proportional to $1 / \sqrt{(U / c)^{2}-1}$ when $U>c$. As the moving speed increases, the water surface elevation first increases and then decreases, and it reaches its peak at $U=c$. It is predicted that a resonance occurs when $U=c$, where the water surface elevation would be infinite. However, it cannot happen actually because of the nonlinear effects.

The dots in Figure 4 are the computed maximum value of water surface elevation in cases of different moving speeds. And the solid lines in Figure 4 denote

$$
\eta / \eta_{0}=\frac{1}{\sqrt{\left|1-(U / c)^{2}\right|}}
$$

Eq.(5) is used as a reference, which is not the original form of Inui's solution. It can be seen that Eq.(5) can well predict the maximum water surface elevation when $U$ is far away from $c$. However, a big deviation will be aroused when $U$ is close to $c$. Obviously, the analysis based on the linear wave theory would fail when the displacement of the water surface is large. The nonlinear effect plays a very important role in the forced wave induced by a pressure disturbance moving with a speed closed to $c$, which may be influenced by the maximum pressure drop and the spatial scale of the pressure disturbance.

\subsection{Effect of the distribution of the low pressure}

In this section, the effects of the maximum pressure drop $\Delta P$ and the radius of the 
pressure disturbance $R_{m}$ on wave pattern are studied. Figure 5 shows the profile of water surface elevation in case of different maximum pressure drop. In those cases, $R_{m}$ is fixed as $40 \mathrm{~km}$ and $U$ is chosen to be $0.5 c, 1.0 c$ and $2.0 c . \Delta P$ varies from $20 \mathrm{hPa}$ to $200 \mathrm{hPa}$. The result shows that the maximum of water surface elevation is approximately in proportion to the maximum pressure drop. The wave pattern remains the same when only the maximum pressure drop is changed.

However, if we check the relative water surface elevation $\eta / \eta_{0}$, a difference among different $\Delta P$ can be found. Figure 6 shows the maximum of water surface elevation varying with low-pressure moving velocity in case of $\Delta P=2 \mathrm{hPa}, 20 \mathrm{hPa}$, 200hPa. In each case, a good agreement between Eq.(5) and the numerical results is found when $U$ is less than $0.5 c$ or larger than $1.5 c$. When $U$ is close to $c$, a significant difference appears where the analytical solution gives an infinite water surface elevation. The computed relative water surface elevation induced by smaller $\Delta P$ shows a higher peak, which is more close to that computed by the linear analytical solution.

Figure 7 shows the profile of water level change along $x=0$ with the variation of $R_{m}$. Here, $\Delta P$ is fixed as $20 \mathrm{hPa}$, and $R_{m}$ varies from $4 \mathrm{~km}$ to $40 \mathrm{~km}$. It is clear that small pressure spatial size is related to small spatial range of water level change. When $U<c$, as shown in Figure7(a), the peaks of water surface elevation are all located at the center of the pressure disturbance. When $U \geq c$, a lag between the peak of water surface elevation and the center of pressure disturbance appears. The lag increases as $R_{m}$ increases.

In both cases that $U=0.5 c$ and $U=2 c$, the peak values of water surface elevation in case of different $R_{m}$ are almost the same. However, a big difference on the peak value of water surface elevation can be noticed in the case that $U=1.0 c$, as shown in Figure7(b). The larger $R_{m}$ gives a smaller peak value of water surface elevation. If we plot the peak value of water surface elevation against $U / c$ in case of different $R_{m}$, as shown in Figure 8, the difference near $U=c$ is more clear. The results shows that the maximum water surface elevation near $U=c$ is also related with the spatial scale of pressure disturbance. A moving low pressure of smaller scale may cause larger water level rise. 


\section{Conclusions}

Numerical simulations on waves induced by a moving pressure disturbance are conducted, based on the nonlinear shallow water equations. The numerical results demonstrate that the wave patterns are greatly affected by the moving speed of the pressure disturbance. When the moving speed of pressure disturbances is less than the phase velocity of shallow water wave, the distribution of water level change is approximately corresponding with the pressure disturbance. When the moving speed of pressure disturbance is larger than the phase velocity of shallow water wave, a triangular wave pattern is observed. Changes on the central pressure drop and the radius of the pressure disturbance do not make pronounced change on the induced wave pattern, but may affect the amplitude of the induced wave.

As the moving speed of pressure disturbance increases, the maximum water surface elevation increases when $U<c$ and decrease when $U>c$. The maximum water surface elevation in each case reaches a peak when $U=c$, where an infinite water surface elevation is predicted by the classic analytical solution. When $U<0.5 c$ or $U>1.5 c$, the central pressure drop and the radius of the pressure disturbance do not make significant influence on the relative maximum water surface elevation. However, when $U$ is close to $c$, the peak of the maximum water surface elevation is significantly affected by the distribution of the pressure disturbance. The numerical results show that a moving low pressure of smaller central pressure drop induces a smaller water surface elevation but a larger relative water surface elevation. Moreover, a moving low pressure of smaller scale may cause larger water level rise.

\section{Acknowledgement}

The authors would like to acknowledge the support by the State Key Laboratory of Hydroscience and Engineering of China under the grant No. 2014-KY-02 and the National Natural Science Foundation of China under the grant No. 51109119 and 51479101.

\section{References}

[1] Inui T. On deformation, wave patterns and resonance phenomenon of water surface due to a moving disturbance. Proc Physico-Mathematical Society of 
Japan, 1936, 18: 60 -113.

[2] Greenspan H P. The generation of edge waves by moving pressure distributions. J Fluid Mech, 1956, 1(6): 574-592.

[3] Abraham G. Modeling study of water gravity waves generated by a moving circular low-pressure area. J Geophys Res, 1963, 68(8): 2185-2210.

[4] Mercer D, Sheng J Y, Greatbatch R J, et al. Barotropic waves generated by storms moving rapidly over shallow water. J Geophys Res-Oceans, 2002, 107(C10).

[5] Vennell R. Long barotropic waves generated by a storm crossing topography. $\mathbf{J}$ Phys Oceanogr, 2007, 37(12): 2809-2823.

[6] Vennell R. Resonance and trapping of topographic transient ocean waves generated by a moving atmospheric disturbance. J Fluid Mech, 2010, 650: 427-442.

[7] Thiebaut S, Vennell R. Resonance of long waves generated by storms obliquely crossing shelf topography in a rotating ocean. J Fluid Mech, 2011, 682: 261-288.

[8] An C, Liu P L F, Seo S N. Large-scale edge waves generated by a moving atmospheric pressure. Theoretical and Applied Mechanics Letters, 2012, 2, 042001.

[9] Seo S, Liu P L F. Edge waves generated by atmospheric pressure disturbances moving along a shoreline on a sloping beach. Coast Eng, 2014, 85: 43-59.

[10]Proudman J. The effects on the sea of changes in atmospheric pressure. Geophysical Journal International, 1929, 2, 197-209.

[11]Monserrat S, Vilibi I, Rabinovich A B. Meteotsunamis: atmospherically induced destructive ocean waves in the tsunami frequency band. Nat Hazards Earth Syst Sci, 2006, 6, 1035-1051.

[12]Niu X, Ueyama S, Sato S, et al. Sediment movement under combined waves, tide and river discharge in a river mouth. Proc Coastal Dynamics 2009, 2009, N0.67. 
Figure 1

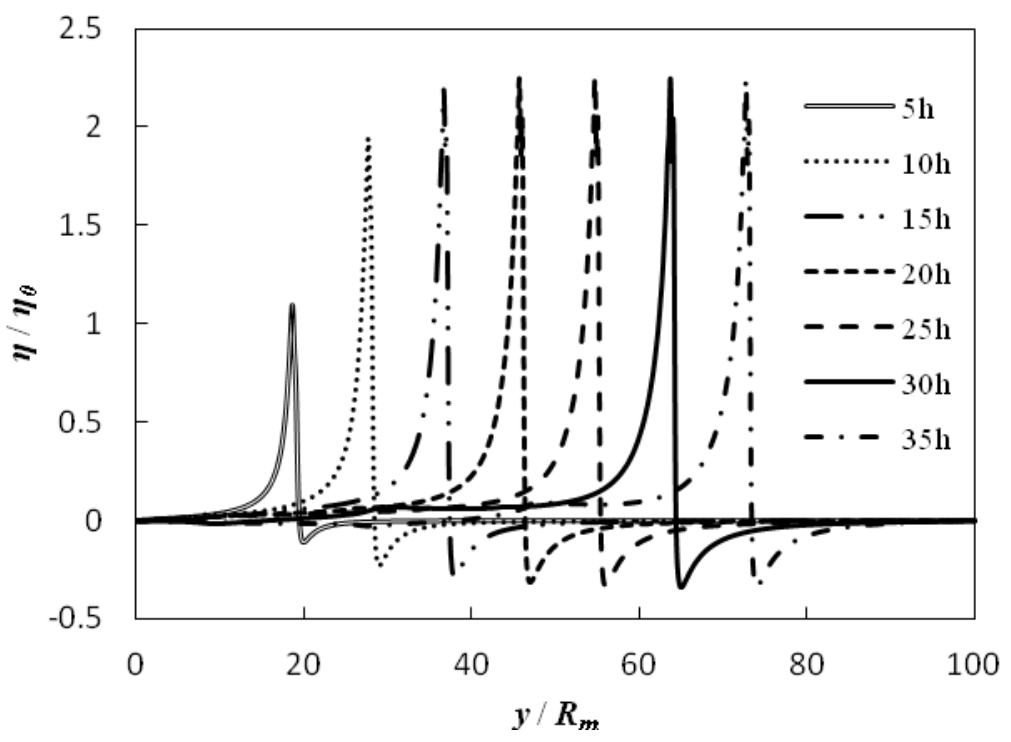

Figure 1 Water surface elevation along $x=0$ in the case of $U=C$ at different time. 


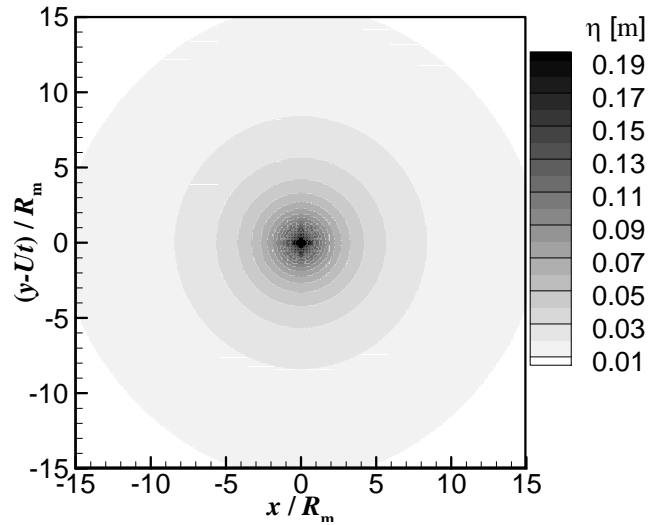

(a) $U=0$

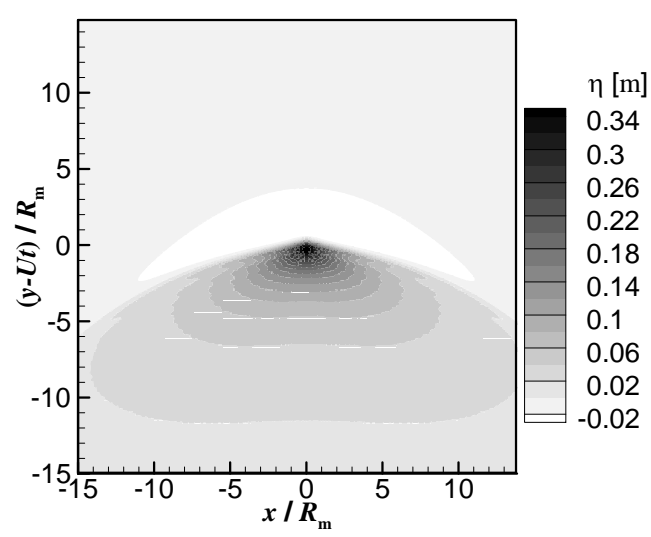

(c) $U=1.0 \mathrm{c}$

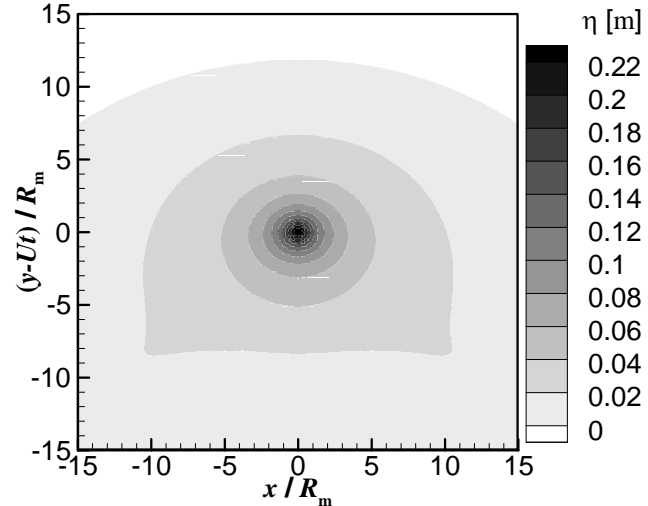

(b) $U=0.5 c$

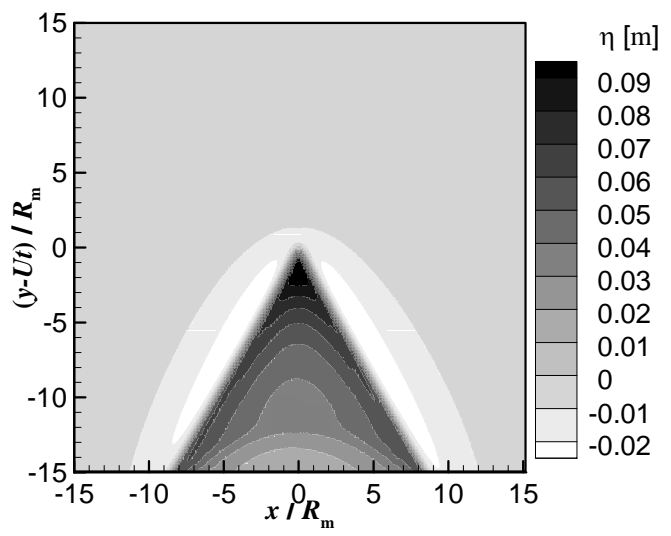

(d) $U=2 c$

Figure 2 Wave pattern induced by the pressure disturbance moving with different speed. 
Figure 3

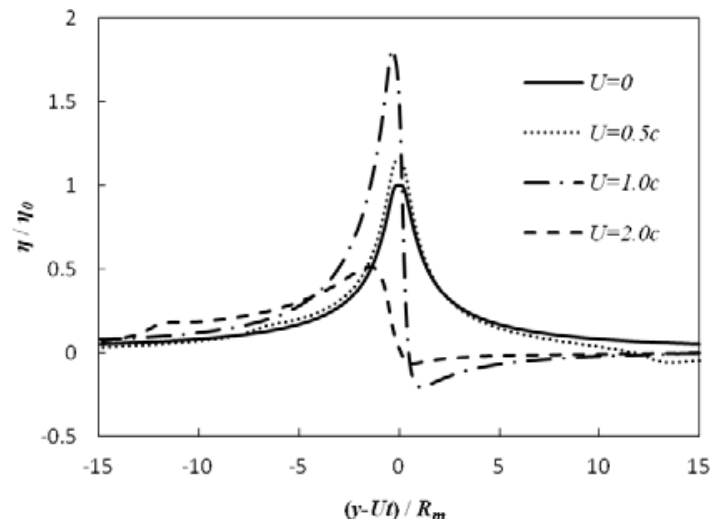

Figure 3 Water surface elevation along the movement direction of low pressure 
Figure 4

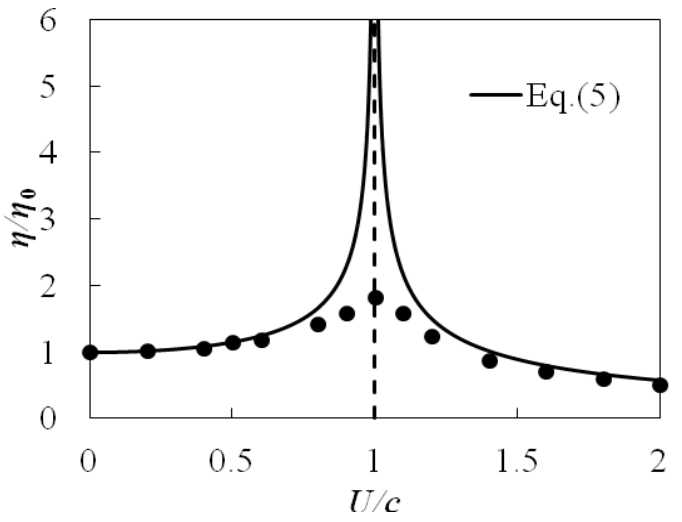

Figure 4 The maximum of water surface elevation varying with low-pressure moving speed 


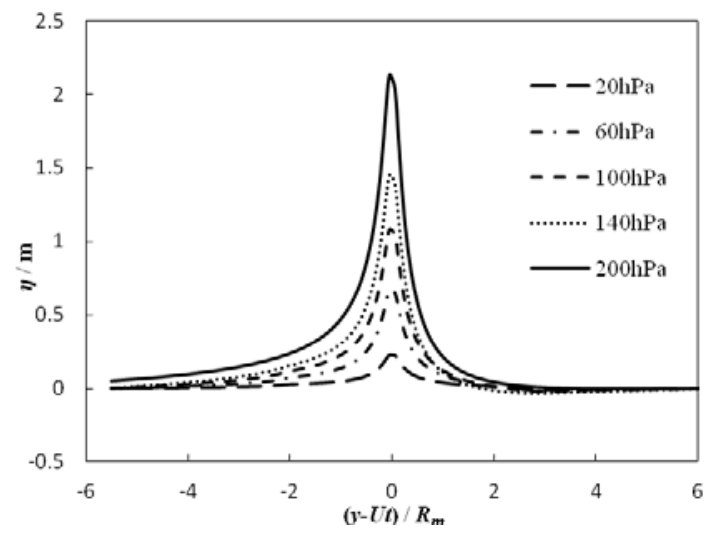

(a) $U=0.5 c$

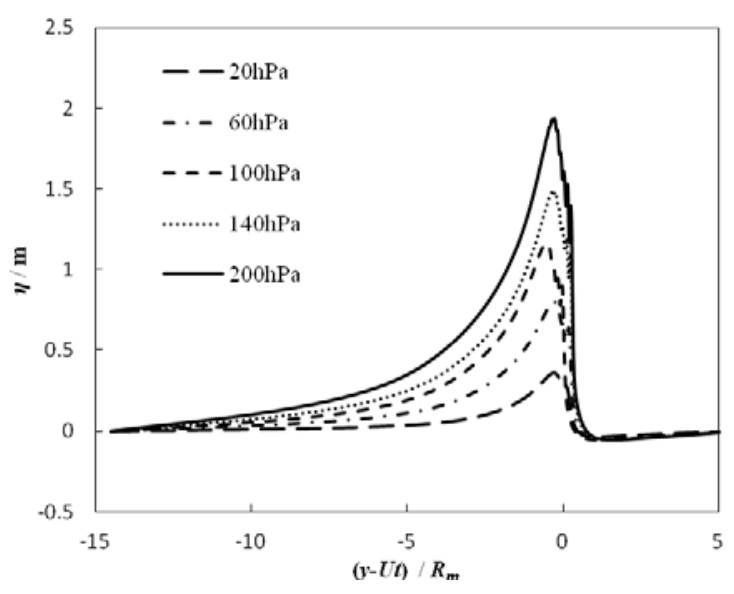

(b) $U=1.0 c$

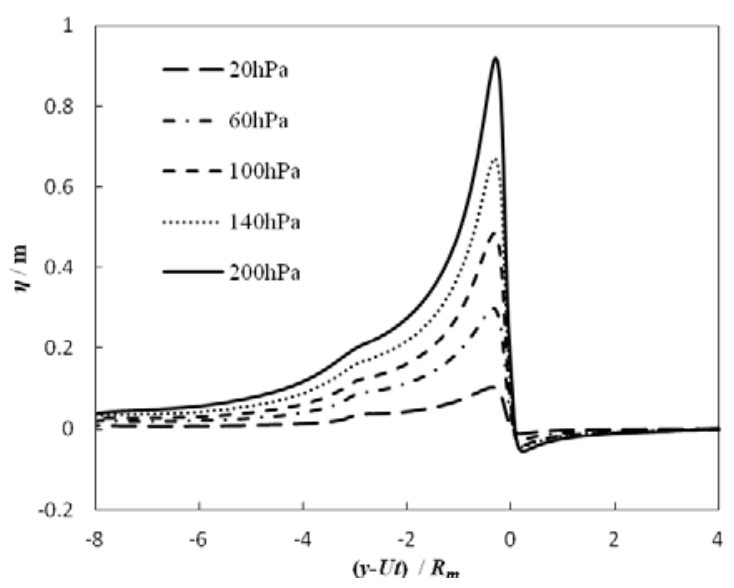

(c) $U=2.0 c$

Figure 5 Water surface elevation varying with the central pressure disturbance 
Figure 6

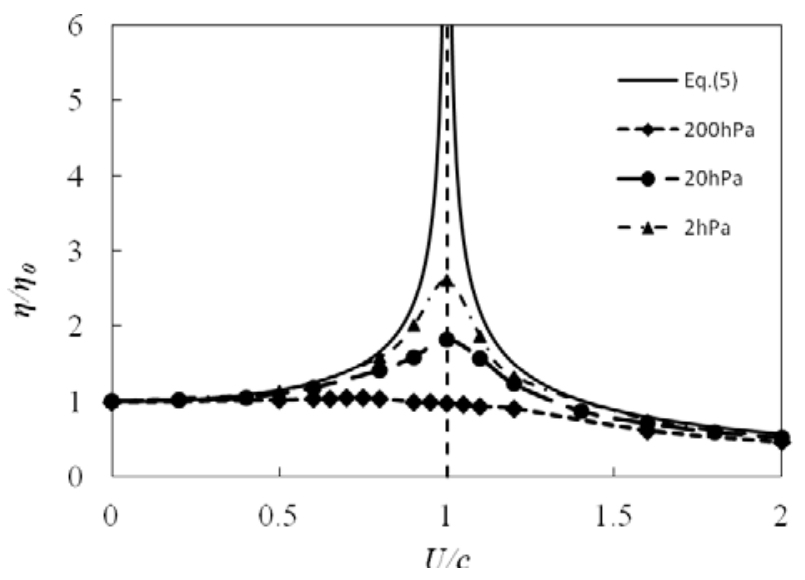

Figure 6 The maximum of water surface elevation varying with low pressure moving velocity in case of different $\Delta P$ 


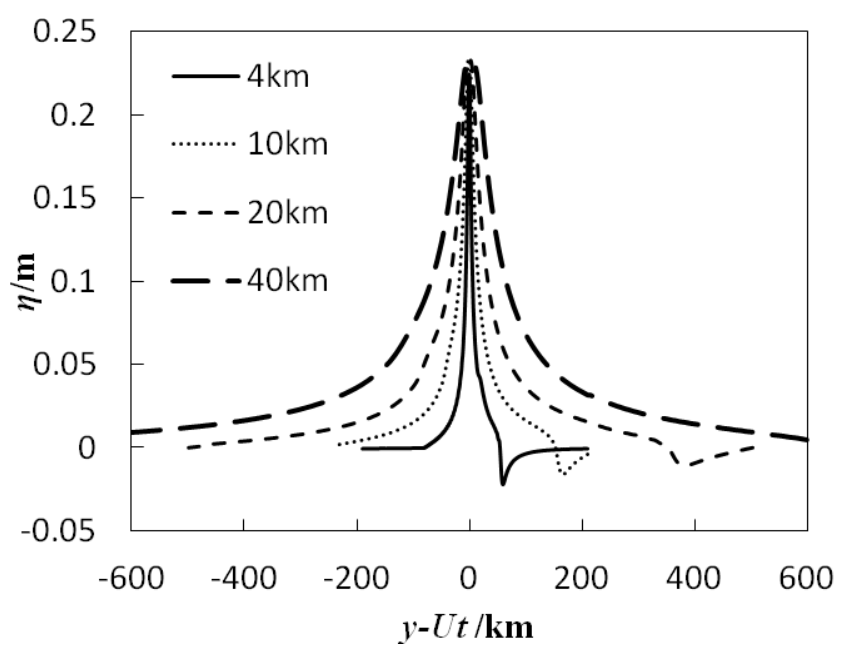

(a) $U=0.5 c$

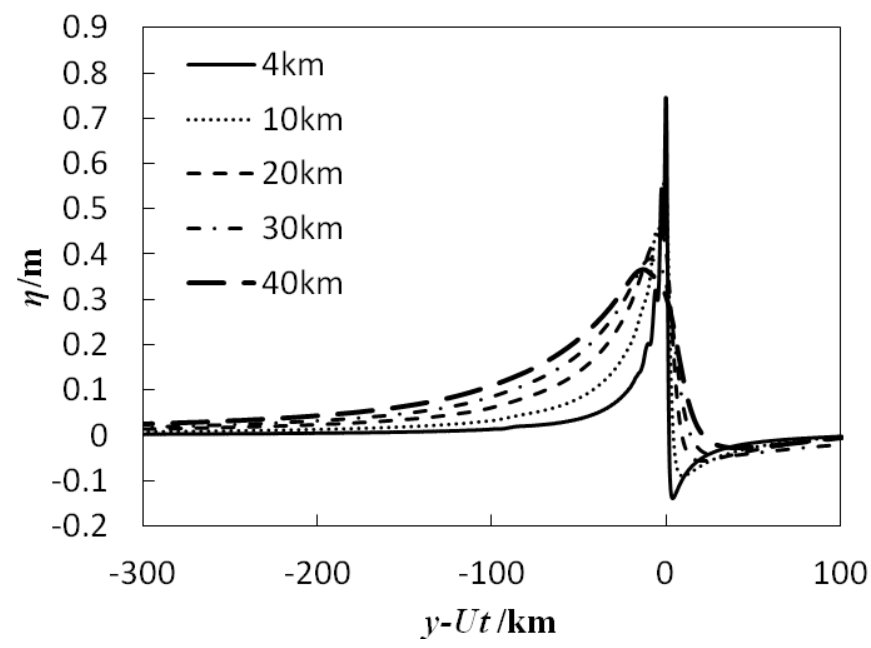

(b) $U=1.0 \mathrm{c}$

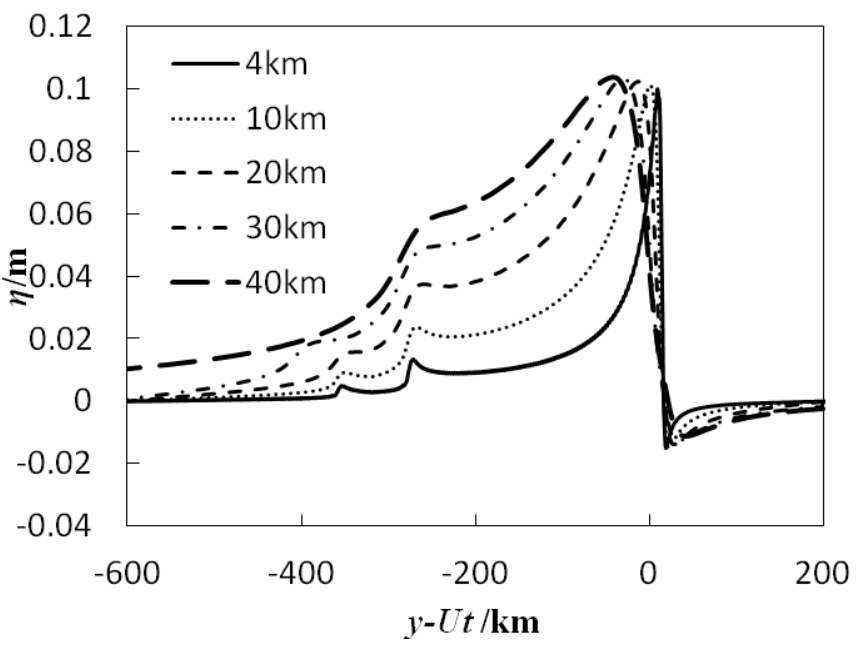

(c) $U=2 c$

Figure 7 Water surface elevation varying with spatial size of the pressure disturbance 
Figure 8

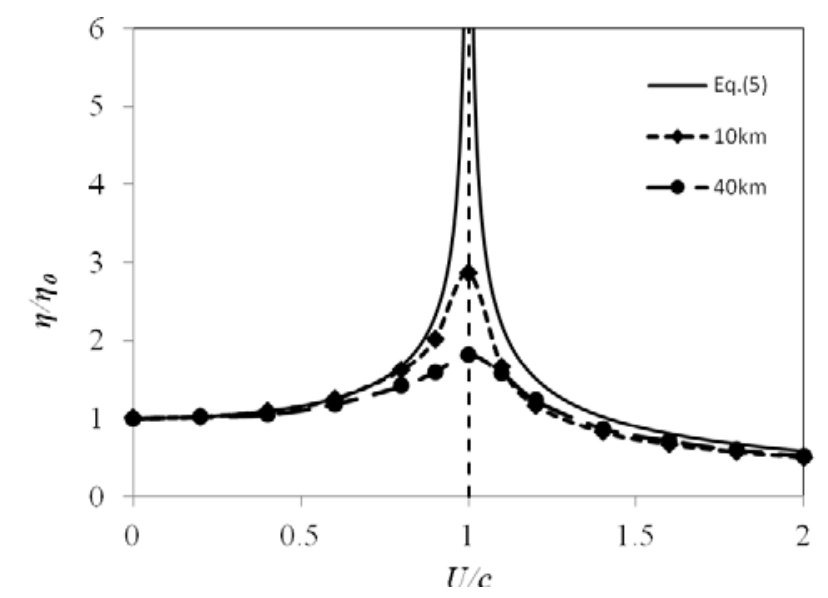

Figure 8 The maximum of water surface elevation varying with low pressure

moving velocity in case of different $R_{m}$ 